\title{
Inflammation in androgenetic alopecia and hair loss: linking neurosciences-endocrinology-dermatology
}

\author{
Marty E. Sawaya, MD, PhD Ocala, Florida, USA mesawaya@att.net
}

The development of androgenetic alopecia (AGA) depends on genetics and androgens, but the specific cellular and molecular events taking place in this multifaceted, complex interaction are vague and unknown. A recent publication now links androgens to inflammation by triggering the innate immune system, which may be a key in triggering the earliest events in the hair cycle as it pertains to men with AGA. ${ }^{1}$ Understanding the specific role that tissue active androgens, such as $5 \alpha$-dihydrotestosterone (DHT) and testosterone, play in shortening the anagen, cycle, from years of growth to months, may lead to discovery of compounds to arrest or prevent the process.

Inflammatory cells surround the hair follicles in scalp biopsies of men with AGA, ${ }^{2}$ especially around the mid to inferior segments of the hair shaft, but it is unknown if androgens influence the inflammatory process and if these earliest inflammatory steps may be a common pathway to all hair signaling pathways that shut down hair growth. There may be common pathways or pathways that overlap in the complicated pathology that takes place in the multitude of hair diseases that affect men, women, and children. Can we learn something from studying these earliest inflammatory events by assessing our current therapies prescribed to men with AGA? The recent publication by De Rivero Vaccari et al. takes a look at how finasteride (Propecia, Merck \& Co) affects hair growth in men with AGA. ${ }^{1}$ This study has shed light on some very important findings that may have relevance across the board to the earliest events taking place in the inflammatory response pathway. This knowledge may be vital in future therapeutic strategies to more effectively treat hair and other skin diseases. These studies also provide links between the neurosciences and endocrinology with dermatology.

In the past 10 years, volumes of publications have linked the innate immune pathway to a host of human diseases, from spinal cord injury to rheumatoid arthritis to infectious diseases, and more. In the last few years, great relevance has linked these earliest steps of the innate pathway to various skin diseases, including psoriasis, UV radiation, vitiligo, contact hypersensitivity, pruritis and, now, hair diseases.

\section{Innate Immunity/Innate Immune Responses}

The pilosebaceous unit is an immunocompetent organ in human skin. Keratinocytes, sebocytes, dermal papilla, and epidermal cells may act as immune cells capable of pathogen recognition. ${ }^{3}$ For example, during an infection, one of the first forms of defense via the innate immune response (IIR) is a group of pattern recognition receptors (PRRs) encoded in the germline to recognize molecular patterns expressed by invading pathogens (Figure 1). ${ }^{4}$ These PRRs can be cytoplasmic, such as Nod-like receptors (NLRs) and RIG-like hilicase receptors (RLRs), or on the membrane surface, such as Toll-like receptors (TLRs) and C-type lectin receptors (CLRs). In 2002, a subset of NLRs named NLRP1 were able to assemble and oligomerize into a common structure that activated the caspase- 1 cascade, leading to the production of pro-inflammatory cytokines, especially IL-1B and

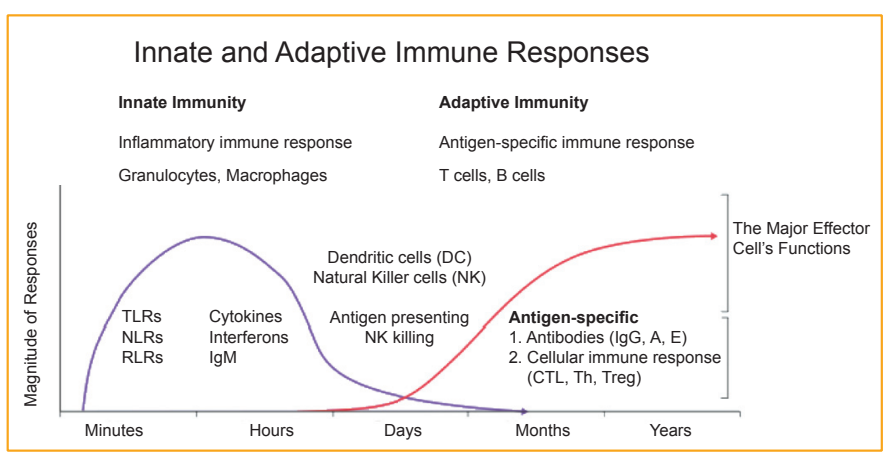

Figure 1: Innate and adaptive immune responses.

IL-18. ${ }^{4}$ This NLRP1 multi-molecular complex was named $I n$ flammasome and, since then, several other inflammasomes have been discovered with two being subsets of NLR: NLRP3 and NLRC4. Recent findings have found an inflammasome termed Absent In Melanoma 2 (AIM2), which assembles upon sensing foreign cytoplasmic double-stranded DNA (dsDNA), which may be important in systemic lupus erythematosis. ${ }^{5}$

Innate immunity molecules or inflammasomes, such as TLRs, are expressed in epidermal and dermal skin cells and, following the pathogen recognition cascade, the pro-inflammatory cytokines are produced and cleaved to active forms of IL-1A, IL-1B, TNF-A, IL-6, IL-8, and others.

If the body is unable to mount this IIR or if the infection or damage is too great, it recruits the Adaptive Immune Response (AIR), which is involved with $\mathrm{T}$ and $\mathrm{B}$ cells further downstream. Figure 1 describes the timing or sequence of events and relationship between IIR and AIR. Recent research is now showing the IIR may be governing the AIR, giving more importance to unraveling these earliest events in inflammation and cell death. ${ }^{6}$

Various other stimuli can induce cytokine production, from microbes to hormones and more, triggering the TLRs or inflammasomes. An essential part of the IIR in humans has been found to be the assembly of these inflammasomes, which are also described as "large scaffolding protein complexes," that activate caspase-1, which is now also thought to affect the downstream AIR inducing further T-cell mediated inflammatory reactions in skin.

In 2002, we analyzed the expression pattern of the critical inflammasome-signaling proteins, such as caspase- 1 , in individuals with AGA and compared to normal scalp skin. ${ }^{7}$ It is well known that inflammation and apoptosis are regulated by an intracellular proteolytic cascade, primarily mediated by the Caspase family of cysteine proteases, of which there are several. Caspases are in a pro-uncleaved form in the cytosol but once activated by signals to the cell (such as death and dying cells, viruses, bacteria, high potassium, ATP, uric acid, etc.) the events for activation of inflammasomes occurs, which then signal the cleavage of Pro-IL1B and Pro IL-8 into active forms via the IIR to start the inflammatory cascade of events. At that time, we had no idea what all of these results meant, but now, 10 years later, the current work makes sense of that earlier work and the 
cascade of events found then is verified again in these current findings. We "had it all the time": the pieces to the puzzle were there from our findings, but we just weren't sure of the relevance of caspase- 1 and being unique to human hair follicles in AGA until now. The studies from 2002 give greater meaning to our recently published work in 2012 by verifying that caspase- 1 is a critical component to IIR and inflammasome activation in the hair follicle, and that inflammation is a very early event that may be a common pathogenetic factor in AGA and other inflammatory hair disorders and diseases.

Studies from 2002 and 2012 take a look at the current oral FDA-approved treatment for AGA, finasteride (Propecia, Merck $\&$ Co), and assess results of caspase-1 in individuals who respond with visible hair growth when taking the drug compared to those who see no hair growth response when taking the drug for 6 months or longer. ${ }^{1,7} \mathrm{We}$ are able to show that AGA involves activation of caspase- 1 in specific areas of scalp, and that finasteride treatment decreases the expression of caspase- 1 in men who have successful hair growth responses. In vitro tissue culture experiments back up these studies revealing that finasteride in human keratinocyte cultures in combinations with and without testosterone, and its associated metabolite, DHT, significantly decreases levels of caspase- $1 .{ }^{1}$

From a historical perspective of over 30 years of research in steroid biochemistry of enzymes, receptors, cofactors, and more, continued results show the importance not only to dermal papilla cells and bulge stem cells, but also to the outer and inner root sheath cells of hair follicles. ${ }^{8}$ Immunohistochemical staining of various steroid-metabolizing enzymes from 5a-reductase type I and II, to the various steroid dehydrogenase/isomerase enzymes, steroid receptors, and now caspase- 1 expression, are found within the cells of the outer and inner root sheaths, giving importance to this area of the hair follicle which encapsulates the entire shaft. ${ }^{1,7,8}$ The amounts of these factors vary depending on the area of scalp, as well as on the sex (men vs. women), various scalp conditions, and body sites of hair. ${ }^{8}$ This gives further support that the hair follicle is a very active unit and that cells from various areas of the follicle contribute to the unique interactive functions vital for the support, function, and continued cycling of hair follicles. Figure 2 shows the immunohistochemical expression of androgen receptors and caspase- 1 expression in the outer and inner root sheaths of human scalp hair follicles (panel $\mathrm{A}$ and $\mathrm{C}$, male patient with AGA treated 2 years with finasteride with significant hair growth response; panel B-androgen receptors and panel D-caspase- 1 the male patient with AGA prior to finasteride therapy). It can be seen that the patient with 2 years of finasteride therapy shows a significant reduction in androgen receptor (A) and caspase-1 (C) expression compared to the start of treatment, panel B and D, respectively.

Inflammasomes have also been found to play a vital role in the IIR to microbial events in the pathogenesis of acne. ${ }^{3}$ With this, androgens have been found to have a vital link to TLR activation, so it makes sense that hair follicles share common pathways to the neighboring gland. Pilosebaceous follicles in acne lesions are surrounded with macrophages expressing TLR2 on their surface, leading to activation of the large scaffolding inflammasome, which leads to the transcription and production of cytokines observed in acne lesions. With androgenic stimulation, neuropeptides, growth factors, and interleukins lead to abnormal ductal and infundibular hyperkeratinization, resulting in inflam-

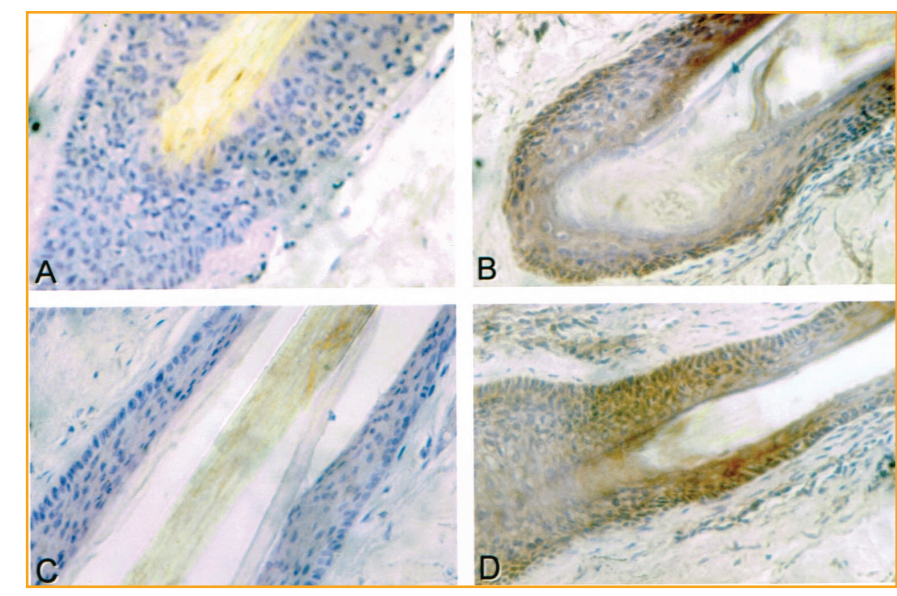

Figure 2: Immunohistochemical expression of androgen receptors and caspase-1 in AGA. Panels $A \& C$ are a male patient with AGA treated for 2 years with finasteride showing decreased to no expression of androgen receptors and caspase-1. Panels $B \& D$ show prior to treatment where significant expression of androgen receptors and caspase- 1 are noted in the outer and inner root sheaths of hair follicles.

mation and rupture of follicular walls and the induction of tissue matrix metalloproteinases that induce scar formation. From these events in acne, could there be some similarity to the cicatricial alopecias? An intriguing thought worth researching.

Sebaceous cells are holocrine, and cells such as these and others have the ability to digest their own organelles and cellular content in a process called "autophagy." Signaling apoptotic events and/or necrosis results in an inflammatory response with certain bacterial pathogens triggering a particular form of cell death mediated by inflammasomes by activation of caspase- 1 . This form of cell death, termed "pyroptosis," combines aspects of both apoptosis and necrosis. The idea of inflammasomes intersecting and signaling with apoptotic machinery illustrates the dual functionality and complexity of this pathway.

Other studies have also found a link to androgens and the inflammasome cascade in the prostate. ${ }^{9}$ Castrated rats show elevated prostate inflammasome TLR protein expression, indicating the ability of testosterone to alter inflammatory cytokine release and modulate key receptors for signaling specific inflammasome IIS responses in vitro as well as in immune competent animal studies, which may represent an important mechanism underlying the immunosuppressive effects that androgens may exhibit.

\section{Nerves to Skin}

IIR has been strongly implicated in abnormal pain hypersensitivity with regard to thermal, mechanical pain sensitivity, and pathological pain whether acute or chronic. ${ }^{10}$ Studies have shown that distinct molecular mechanisms underlie pain and itching, indicating that TLR-7 is expressed in C-fiber primary sensory neurons, which are important for pruritis. Even non-neuronal cells in the skin, such as keratinocytes and mast cells, may play a role in TLR-7-mediated pruritis along with inflammation associated with psoriasis as well as chronic itch resistant to antihistamine treatment. ${ }^{10}$ Thus, targeting TLR-7 may be a promising route for anti-itch treatments for skin in the future.

A lot is known about the AIR including neuropeptides being released from cutaneous nerves or skin and the resulting immune cell response to these stimuli. ${ }^{11}$ Peripheral or cutaneous nerves have been described as being either "afferent," controlling eccrine functions, blood flow, and hair erection to CNS, or "effer- 
ent," transmitting sensory signals from the CNS to effector cells in the peripheral tissues. ${ }^{11}$ However, the discovery of substance $\mathrm{P}$ in sensory nerve fibers leading to other sensory neuropeptides found in skin adds to the complexity of health and disease. A great review of AIR in cutaneous biology is given in a review with a continuing theme of the "brain-skin connection" revealing the complex interrelationships between the organ systems that take place as we uncover more relevance to the importance of IIR to AIR, that the endocrine, nervous, and immune systems interact and that a whole new arena of therapies can now be targeted with utilizing the IIR and the inflammasome pathway. ${ }^{11}$

\section{Future Therapeutics Targeting the Inflammasome Pathway}

Our research fount that both in vivo and in vitro finasteride treatments lower caspase-1 expression in men with AGA, and that androgens influence IIR in the hair cycle. ${ }^{1}$ From what we know from the volumes of research in the neurosciences and other fields, IIR may be the earliest of events taking place in signaling the downstream responses from the AIR in hair diseases. This is an attractive idea to consider because we may be able to diagnostically reveal if the expression of caspase- 1 is elevated prior to miniaturization of the hair follicle so that arresting this elevation at its earliest stages may be a unique way to treat AGA.

Indeed, we have learned a lot from spinal cord paralysis and patients who have suffered from acute spinal cord injury such as Buffalo Bills Kevin Everett. ${ }^{14}$ Treating Everett at the earliest moment of injury resulted in arresting the process of paralysis. Everett recovered from the C3-C4 spinal lesion (same as Christopher Reeve, Mark Buoniconti, and common to other athletes). The combination of hypothermia and high dose, potent corticosteroids found from animal studies successfully arrested the inflammatory process and led the way for validating the importance of inflammasomes and the IIR. ${ }^{12}$ The goal is to find unique modulators, inhibitors of these earliest events that take place within minutes to hours (x-axis) of injury in the IIR (Figure 1) so that irreversible nerve damage does not take place and lead to apoptosis/necrosis.

TLRs and the various inflammasomes found to date have been recognized as therapeutic targets for the development of site-specific treatments. Engagement of TLR ligands by their receptors leads to cytokine production, induction of IIR, and the development of AIR. TLR agonists are being tested for allergic and infectious diseases as well as cancer and HIV. ${ }^{13} \mathrm{~A}$ trial using TLR3 ligand poly-AU showed improvement in a 20-year survival study in a subset of breast cancer patients with tumor cells that expressed TLR3. ${ }^{13}$

Therapeutic approaches targeting inflammasome products for autoimmune disorders have shown that anakinra, a recombinant human IL-1 receptor antagonist, produced a favorable response in rheumatoid arthritis in the 2,846 patients studied. ${ }^{13}$ Neutralizing the NLRP1 inflammasome reduces the severity of thromboembolic strokes in rodent models and traumatic brain injury associated with inflammation. ${ }^{13}$ A caspase- 1 inhibitor, VRT, has been reported to reduce brain damage in animal models of transient cerebral ischemia.

Future studies and development of therapies involving IIR is beyond the scope of this report as the literature is filled with a vast amount of compounds, regulators, activators, inhibitors, genetic targets, and more to the IIR pathways for various human diseases. It's obvious to see from the success of spinal cord injury patients like Kevin Everett that finding new and novel compounds to affect IIR, and specifically inflammasomes, may eventually be used alone or in combination with our current AIRrelated therapies to optimize nerve cell integrity and function to minimize the inflammatory and apoptotic events leading to permanent paralysis. If these agents are found, it is certain they will be assessed for other indications, and it is believed that they will hold great promise in hair and skin diseases.

\section{References}

1. De Rivero Vaccari, J.P., et al. Caspase-1 level is higher in the scalp of androgenetic alopecia. Dermatol Surg. 2012; 1-7 online.

2. Whiting, D.A. Color atlas of differential diagnosis of hair loss. Cedar Grove, NJ: Canfield Publishing, 1996.

3. Kurokawa, I., et al. New developments in our understanding of acne pathogenesis and treatment. Experimental Dermatol. 2009; 18:821-832.

4. Conforti-Andreoni, C., et al. The inflammasomes in health and disease: from genetics to molecular mechanisms of autoinflammation and beyond. Cellular \& Molec Immunol. 2011; 8:135-145.

5. Dombrowski, Y., et al. Cytosolic DNA triggers inflammasome activation in keratinocytes in psoriatic lesions. www.sciencetranslationalmedicine.org. 11 May 2011; 3(82):82ra38.

6. Kufer, T.A., and P.J. Sansonetti. NLR functions beyond pathogen recognition. Nature Immunol. 2011; 12(2):121-128.

7. Sawaya, M.E., et al. Effects of finasteride on apoptosis and regulation of the human hair cycle. J Cutan Med Surg. 2002; 6:1-9.

8. Sawaya, M.E., and V.H. Price. Different levels of $5 \alpha$-reductase type I and II, aromatase and androgen receptor in hair follicles of women and men with AGA. J Invest Dermatol. 1997; 109:296-300.

9. Rettew, J.A., Y.M. Huet-Hudson, and I. Marriott. Testosterone reduces macrophage expression in the mouse of toll-like receptor 4, a trigger for inflammation and innate immunity. Biol Reproduction. 2008; 78:432-437.

10. Liu, T., et al. Toll-like receptor 7 mediates pruritis. Nature Neurosci. 31 October 2010; online.

11. Peters, E.M., et al. Neuropeptide control mechanisms in cutaneous biology: physiological and clinical significance. J Invest Dermatol. 2006; 126:1937-1947.

12. De Rivero Vaccari, J.P., et al. A molecular platform in neurons regulates inflammation after spinal cord injury. $J$ Neurosci. 2008; 28(13):3404-3414.

13. Yu, M., and S.J. Levine. Toll-like receptor 3, RIG-1-like receptors and the NLRP3 inflammasome: key modulators of innate immune responses to double-stranded RNA viruses. Cytokine \& Growth Factor Reviews. 2011; 22:63-72.

14. Kevin Everett: NFL's Everett talks about his recovery from paralysis. Tuesday, October 12, 2010. www.abclocal. go.com. 